\title{
Laparoscopic nephroureterectomy in a patient with a left ventricular assist device
}

\author{
Jasmir G. Nayak, MD; $;^{*}$ Christopher W. White, MD; ${ }^{\dagger}$ Wayne Nates, MD; $;$ Rajan Sharda, MD; ${ }^{*}$ David Horne, MD; ${ }^{\dagger}$ \\ Kam Kaler, MD; ${ }^{*}$ Mark Lytwyn, MD; ${ }^{\dagger}$ Hilary P. Grocott, MD; Darren H. Freed, MD; Thomas Mcgregor, MD, FRCSC
}

*Section of Urology, Department of Surgery, University of Manitoba, Winnipeg, MB; †Section of Cardiac Surgery, Department of Surgery, University of Manitoba, Winnipeg, MB; §Department of Anesthesia, University of Manitoba, Winnipeg, MB

Cite as: Can Urol Assoc J 2013;7(9-10):e640-4. http://dx.doi.org/10.5489/cuaj.400

Published online October 9, 2013.

\section{Abstract}

Left ventricular assist device (LVAD) therapy is an established treatment option for select patients with advanced heart failure. Advances in technology and patient management have resulted in improved post-implant outcomes. Consequently, more patients with LVADs are presenting for evaluation and care of non-cardiac surgical disease. However, there is a paucity of literature regarding the optimal perioperative and surgical management of such patients. We present the case of a 71-year-old male with a HeartMate II (Thoratec Corporation, Pleasanton, CA) LVAD, who underwent a laparoscopic left nephroureterectomy for an upper urinary tract transitional cell carcinoma. His perioperative course was uneventful due to the multidisciplinary efforts of cardiac surgery, cardiac anesthesia, nephrology and urology. To our knowledge, this is the first reported case of a laparoscopic nephroureterectomy in a patient with a HeartMate II LVAD.

\section{Background}

Mechanical circulatory support with a left ventricular assist device (LVAD) is an established therapeutic option for select patients with advanced heart failure, as either a bridge to transplantation ${ }^{1}$ or for permanent support. ${ }^{2}$ Technological advances and improvements in patient management have resulted in improved patient outcomes ${ }^{2-4}$ and have led to an increasing number of patients being supported with a LVAD. ${ }^{5}$ A substantial proportion of these patients require surgical management of non-cardiac disease. ${ }^{6}$ The upper abdominal, pre-peritoneal location of the HeartMate II (HMII) LVAD (Thoratec Corp, Pleasanton, CA) precludes extension of median or paramedian incisions (Fig. 1) $;$ however, literature regarding the safety and feasibility of laparoscopic surgery in patients supported with this device is limited (Table 1).
Upper urinary tract transitional cell carcinoma (TCC) is an aggressive malignancy in which surgical intervention is the standard therapy to achieve adequate oncological control. There is growing interest in nephron-sparing techniques, including endoscopic approaches and segmental resection; however, the oncological efficacy of these treatments has not been definitively established. Consequently, complete surgical excision by radical nephroureterectomy remains the standard of care, and the laparoscopic approach is associated with less blood loss, smaller incisions, reduced postoperative pain, and a shorter hospital length of stay. ${ }^{7}$ The laparoscopic approach is especially appealing in patients supported with a HMII LVAD, facilitating safe resection by improving visualization and allowing the placement of incisions remote from the device hardware. ${ }^{8}$

We report on a 71-year-old male with a HMII LVAD and a history of upper urinary tract TCC with refractory hematuria that was successfully treated with a laparoscopic nephroureterectomy.

\section{Case presentation}

A 71-year-old male, with chronic kidney disease (CKD) and an ischemic cardiomyopathy that required implantation of a HMII as a bridge to transplant candidacy in 2009, initially presented in 2002 for urologic assessment of gross hematuria. Cystoscopy at the time revealed a bladder lesion that was resected, with subsequent histologic analysis revealing a low-grade papillary lesion and carcinoma in situ. He was treated with an induction course of intravesical Bacillus Calmette-Guérin immunotherapy; however, in early 2010 he developed a recurrent low-grade bladder lesion and distal left ureteric lesion. In light of the patient's significant cardiac comorbidities, both of these lesions were managed endoscopically. Subsequent follow-up was negative for recurrence until the patient developed painless gross hematuria in late 2010. Retrograde pyelography suggested the presence of a left lower pole filling defect (Fig. 2) and flexible ure- 


\begin{tabular}{lccc}
\hline Table 1. Reported cases of laparoscopic abdominal surgery in patients supported with a LVAD \\
\hline Author & No. patients & Type of LVAD & Surgery \\
Prendergast et al. $1996^{11}$ & 1 & ABIOMED & BVS 5000 \\
& 1 & BiVAD & Diagnostic laparoscopy \\
Nissen et al. $2005^{12}$ & 1 & Thoratec BiVAD & Laparoscopic cholecystectomy \\
Kartha et al. $2008^{13}$ & 5 & Not specified & Laparoscopic cholecystectomy \\
Groth et al. $2008^{14}$ & 2 & Novacor LVAD & Laparoscopic diaphragmatic hernia repair \\
Livi et al. $2009^{15}$ & & Berlin Heart Excor LVAD & Laproscopic colon resection \\
& 1 & Heartmate II LVAD & Laparoscopic cholecystectomy \\
Brown et al. $2009^{16}$ & 1 & Impella LP2.5 LVAD & Laparoscopic cholecystectomy \\
Atoui et al. $2010^{17}$ & 1 & Novacor LVAD & Laparoscopic splenectomy \\
Bennet et al. $2010^{18}$ & 2 & Heartmate II LVAD & Laparoscopic colon resection \\
Stewart et al. $2011^{8}$ & 1 & Heartmate II LVAD & Laparoscopic splenectomy \\
Samoukovic et al. $2011^{19}$ & 1 & Heartmate II LVAD & Laparoscopic gastric bypass \\
Hoefnagel et al. $2012^{20}$ & 1 & Hearmate II LVAD & Laparoscopic colon resection \\
Sathishkumar et al. $2012^{21}$ & 1 & Not specified &
\end{tabular}

terorenoscopy confirmed the presence of a papillary lesion; however, due to poor visualization, biopsy and fulguration could not be performed. The patient's requirement for therapeutic anti-coagulation precipitated recurrent episodes of gross hematuria requiring hospital admissions for continuous bladder irrigation and blood transfusion. The significant morbidity related to these episodes prompted consideration of a laparoscopic left nephroureterectomy. Nephron-sparring options were discussed; however, it was felt that nephroure-

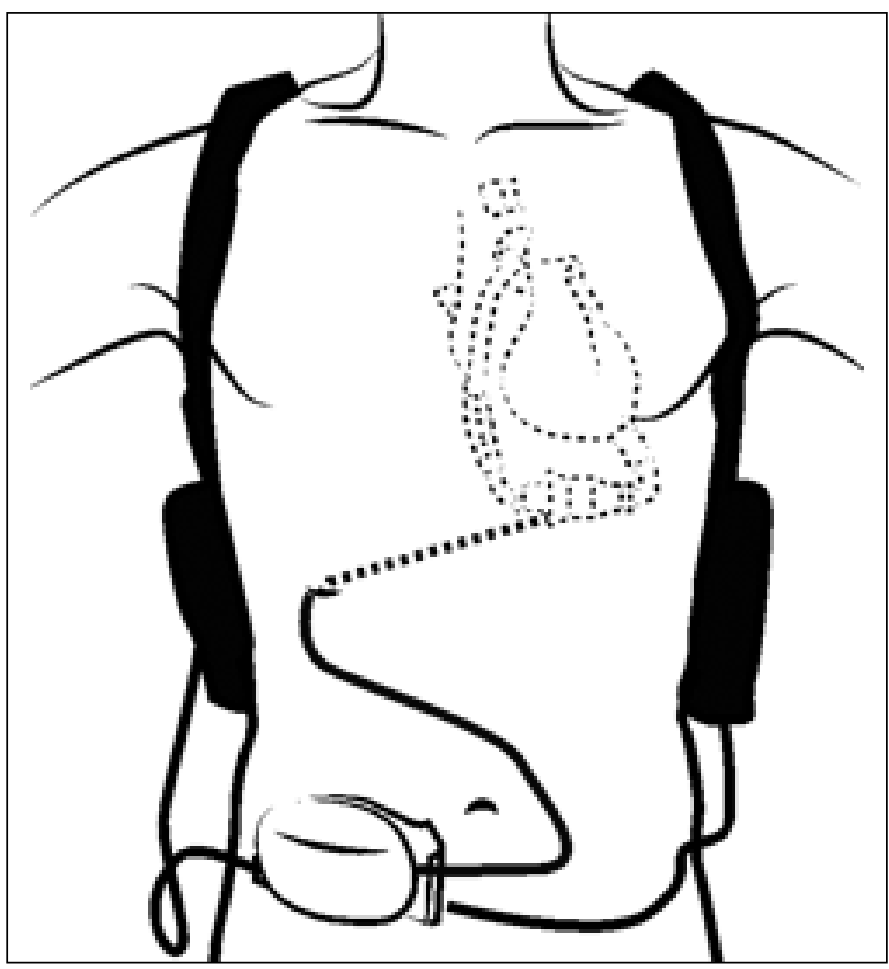

Fig. 1. Heartmate II left ventricular assist device. terectomy would provide the most definitive management. Subsequently, consultation with cardiac anesthesia, cardiac surgery, and nephrology were arranged to assist with preoperative planning and the acquisition of informed consent.

The patient was admitted preoperatively to cardiac surgery to facilitate optimal preoperative management. Due to his CKD (baseline serum creatinine $170 \mathrm{mmol} / \mathrm{L}$ ), a nuclear renogram was performed to delineate the differential function. This revealed $40 \%$ function on the left and $60 \%$ on the right. HMII evaluation demonstrated normal function. Oral warfarin therapy was stopped 3 days prior to surgery and he was subsequently bridged with intravenous heparin. His preoperative international normalized ratio (INR) and partial thromboplastin time (PTT) were 1.4 and $43.1 \mathrm{sec}-$ onds, respectively.

In the preoperative holding area, external defibrillator pads were placed before the anti-tachycardic and defibrillator functions of his implanted cardiac defibrillator device were deactivated. On arrival in the operating theatre, the LVAD was connected to the power-base unit, which displayed continuous LVAD pump flow, pump speed, and pulse index.

The patient was positioned with care to avoid pressure on the driveline and device. A left radial arterial cannula was inserted with ultrasound guidance, and prophylactic antibiotics were administered with the induction of anesthesia. Following endotracheal intubation, a transesophageal echocardiography (TEE) probe was placed. An 8.5 Fr cordis sheath was then inserted into the right internal jugular vein for central venous pressure monitoring and intravenous fluid administration. Surface ultrasonography was used to delineate the anatomic position of the HMII driveline (Fig. 3).

A rigid cystoscope was inserted to circumferentially resect and fulgurate the left ureteric orifice in the standard 


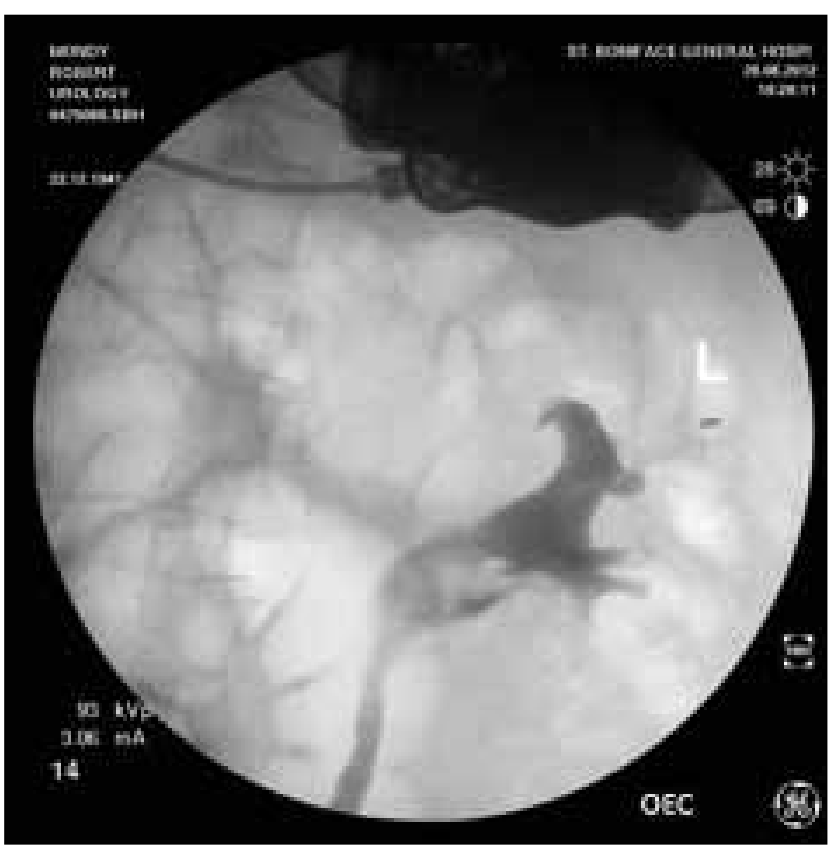

Fig. 2. Left retrograde pyelography demonstrating significant filling defect within renal pelvis.

transurethral fashion. There was no evidence of any bladder lesions. The patient was then repositioned into the right lateral decubitus position. Pneumoperitoneum was achieved using an open Hasson technique, lateral to the umbilicus. The abdomen was slowly insufflated to $12 \mathrm{mmHg}$ and all the remaining port sites were placed under direct vision (Fig. 3). We began by taking down the bowel along the White line of Toldt to displace the bowel medially. The ureter was dissected distally to facilitate the ureterectomy component of the procedure. The absence of arterial pulsations due to the continuous flow nature of the HMII LVAD necessitated meticulous surgical dissection to identify the iliac vessels, while adequately mobilizing the distal ureter. The ureter and gonadal vessel were then followed superiorly to the renal hilum. The renal vein was identified early followed by the renal artery after careful dissection. The single artery and then vein were clipped and stapled accordingly. The superior and lateral renal attachments were then taken down. Once the resected kidney was free, it was placed in an endocatch bag, and a Gibson incision was made to both extract the specimen and complete the distal ureterectomy (Fig. 3). A Jackson-Pratt (JP) drain was placed. Total blood loss for the procedure was less than $50 \mathrm{~mL}$.

Hemodynamic perturbations occurred at various stages of the procedure and were precipitated by decreases in systemic vascular resistance that led to a fall in the HMII LVAD pulse index, leftward septal bowing, left ventricular collapse, dilation of the right atrium and ventricle visible on transesophageal echocardiography (TEE). Small doses (50$100 \mathrm{ug}$ ) of phenylephrine rapidly reversed these changes.
Pneumoperitoneum was otherwise well-tolerated. At the end of the procedure, the patient was extubated and the HMII was reconnected to external battery power. The patient was then transferred to the cardiac surgical intensive care unit for postoperative monitoring and care.

The patient had an uneventful postoperative course. Intravenous heparin was resumed on postoperative day (POD) 1 and oral warfarin on POD 3. The JP drain was removed on POD 4. A Foley catheter was left in situ and removed after 1 week. Postoperative renal function remained stable and the patient was discharged on POD 7. At the 6-week follow-up, the patient was doing well and had resumed regular activities. The final pathology report confirmed the presence of a pT2 high grade TCC, with negative margins.

\section{Discussion}

Advances in technology and patient management have resulted in improved post-LVAD implant outcomes ${ }^{2-4}$ and have led to an increasing number of patients with a LVAD presenting with non-cardiac surgical disease. ${ }^{6}$ Although open partial ${ }^{9}$ and open radical nephrectomy ${ }^{10}$ have been described, this is the first report of laparoscopic renal surgery in a patient supported with an HMII LVAD. Although there is growing interest in less invasive, nephron-sparing techniques, their efficacy is not established and nephroureterectomy continues to serve as the gold standard for the treatment of upper tract TCC.

The HMII LVAD is a continuous flow device implanted in a pre-peritoneal pocket, with inflow and outflow cannulae anastomosed to the left ventricular apex and ascending aorta, respectively. Blood exposure to the foreign surfaces within the HMII LVAD necessitates anti-coagulation with warfarin (INR: 1.5-2.5). Inadequate anti-coagulation may lead to thrombus formation and places the patient at risk for devastating thromboembolic complications and device failure. Conversely, hemorrhage requiring allogeneic blood transfusion increases the risk of immunologic sensitization and makes finding an appropriately matched donor organ for heart transplantation difficult. In this case, all conservative measures to manage the patient's ongoing hematuria had been exhausted and surgical intervention became necessary.

Power to the HMII is supplied via an electrical drive-line tunneled in the anterior abdominal wall (Fig. 1). Injury or fracture of the drive-line could lead to catastrophic pump failure. It is essential to delineate the LVAD drive-line to avoid inadvertent injury when placing ports for laparoscopic surgery. This can easily be achieved with preoperative surface ultrasonography. With the patient in the right lateral decubitus position, the external portion of the drive-line was safely excluded from the surgical field.

The lack of arterial pulsation is an important attribute characteristic of patients supported with a continuous flow 


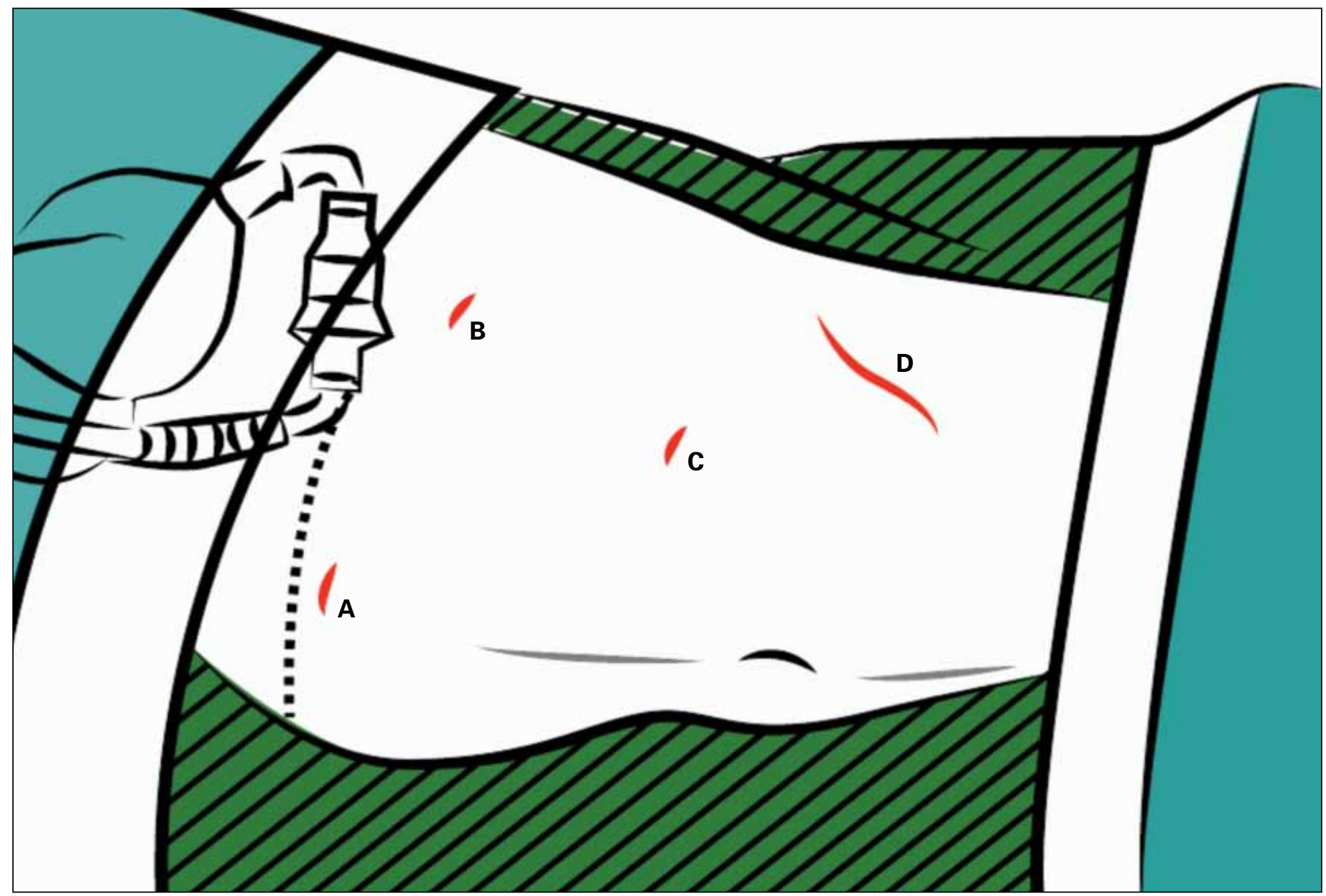

Fig. 3. Patient in right lateral decubitus position with port and extraction sites (in red) for nephroureterctomy identified and anatomic location of the HMII LVAD and driveline superimposed. (A) 5-mm assistants port; (B) 5-mm surgeons left hand port; (C) 10-mm camera port and (D) 10-mm surgeons right hand port which was extended into Gibson incision for extraction of specimen.

LVAD. Although meticulous dissection around the renal hilum is assumed, the visualization of arterial pulsation can often help guide the dissection and identification of the renal artery. However, this is not possible in a patient supported with a HMII LVAD.

An anesthesiologist familiar with the HMII LVAD and able to perform and interpret TEE is essential in the surgical management these patients. Hemodynamic disturbances can be sudden and require swift intervention. Patients with a HMII LVAD are inherently preload dependent, since the device is unable to compensate by decreasing forward flow when preload is reduced. Consequently, the degree of abdominal insufflation must be carefully balanced between visualization and the reduction of venous return. Patients with a HMII are also sensitive to changes in left-ventricular afterload. Decreases in afterload may cause a suction effect and lead to left ventricular collapse, leftward bowing of the inter-ventricular septum, and acute right ventricular failure. This is associated with a rise in central venous pressure and a decrease in mean arterial pressure. Increasing left ventricular afterload with a direct alpha agonist rapidly reverses these changes. The mid-esophageal 4-chamber view of the heart is extremely valuable in interpreting acute hemodynamic changes and ensuring appropriate therapy is instituted in a timely fashion.

Hematuria is one of the hallmark signs of urothelial carcinoma, and can be exacerbated by coagulopathic states. Not only can this cause significant patient morbidity, but it also limits the utility of endoscopic and percutaneous approaches, should they be considered. Due to their need for anti-coagulation, patients with a LVAD should be bridged with intravenous heparin up until the time of surgery, and resumed as soon as possible postoperatively when the risk of postoperative hemorrhage is reduced.

\section{Conclusion}

Laparoscopic renal surgery is feasible and safe, when undertaken in multidisciplinary fashion with input from urology, cardiac surgery, cardiac anesthesia, critical care, and nephrology. 
Nayak et al.

Competing interests: None declared.

This paper has been peer-reviewed.

\section{References}

1. Miller LW, Pagani FD, Russell SD, et al. Use of a continuous-flow device in patients awaiting heart transplantation. N Engl J Med 2007;357:885-96.

2. Slaughter MS, Rogers JG, Milano CA, et al. Advanced heart failure treated with continuous-flow left ventricular assist device. N Engl J Med 2009;361:2241-51.

3. Aaronson KD, Slaughter MS, Miller LW, et al. Use of an intrapericardial, continuous-flow, centrifugal pump in patients awaiting heart transplantation. Circulation 2012;125:3191-200.

4. White CW, Chelvanathan A, Zieroth S, et al. Can Long-Term Ventricular Assist Devices Be Safely Implanted in Low-Volume Non-Heart Transplant Centres? Can J Cardiol 2013;29:983-9. http://dx.doi.org/10.1016/i. cica.2012.07.012. Epub 2012 Sep 26.

5. Stehlik J, Edwards LB, Kucheryavaya AY, et al. The registry of the international society for heart and lung transplantation: 29th official adult heart transplant report-2012.J Heart Lung Transplant 2012;31:105264. http://dx.doi.org/10.1016/i.healun.2012.08.002

6. Stehlik J, Nelson DM, Kfoury AG, et al. Outcome of noncardiac surgery in patients with ventricular assist devices. Am J Cardiol 2009;103:709-12. http://dx.doi.org/10.1016/i.amicard.2008.11.021

7. Rai $B P$, Shelley $M$, Coles $B$, et al. Surgical management for upper urinary tract transitional cell carcinoma (UUT-TCC): a systematic review. BJU Int 2012;110:1426-35. http://dx.doi.org/10.1111/i.1464410X.2012.11341.x. Epub 2012 Jul 3.

8. Stewart DB, Pae WE, Stephenson ER Jr. Laparoscopic colectomy is feasible in patients with left ventricular assist devices. Int J Colorectal Dis 2011;26:1223-4. hittp://dx.doi.org/10.1007/s00384-010-1103-6

9. Manger JP, Kern JA, Krupski TL. Partial nephrectomy in a patient with a left ventricular assist device. Case Rep Urol 2011;2011:526903. Epub 2011 Sep 22.

10. Goldstein DJ, Mullis SL, Delphin ES, et al. Noncardiac surgery in long-term implantable left ventricular assistdevice recipients. Ann Surg 1995;222:203-7. http://dx.doi.org/10.1097/00000658-19950800000013

11. Prendergast TW, Ortega AE, Starnes VA, et al. Safe application of diagnostic laparoscopy during biventricular assistance. Ann Thorac Surg 1996;61:735-7. http://dx.doi.org/10.1016/0003-4975(95)00862-4
12. Nissen NN, Korman J, Kleisli T, et al. Laparoscopic cholecystectomy in a patient with a biventricular cardiac assist device. JSLS 2005;9:481-4.

13. Kartha V, Gomez W, Wu B, et al. Laparoscopic cholecystectomy in a patient with an implantable left ventricular assist device. Br J Anaesth 2008;100:652-5. http://dx.doi.org/10.1093/bja/aen052

14. Groth SS, Whitson BA, D'Cunha J, et al. Diaphragmatic hernias after sequential left ventricular assist device explantation and orthotopic heart transplant: early results of laparoscopic repair with polytetrafluoroethylene. J Thorac Cardiovasc Surg 2008;135:38-43. http://dx.doi.org/10.1016/i.jtcvs.2007.09.017

15. Livi U, Guzzi $G$, Turi $V$, et al. Laparoscopic approach for urgent abdominal surgery in patients with left ventricular assist devices. J Cardiovasc Med (Hagerstown) 2009;10:741-4. http://dx.doi.org/10.2459/ JCM.0b013e328329cbof

16. Brown JB, Hallinan WM, Massey HT, et al. Does the need for noncardiac surgery during ventricular assist device therapy impact clinical outcome? Surgery 2009;146:627-33. http://dx.doi.org/10.1016/i. surg.2009.06.033

17. Atoui R, Samoukovic G, Al-Tuwaijir F, et al. The use of the Impella LP 2.5 percutaneous microaxial ventricular assist device as hemodynamic support during high-risk abdominal surgery. J Card Surg 2010;25:23840. http://dx.doi.org/10.1111/j.1540-8191.2009.00951.x

18. Bennett SK, Sheridan B, Meyers MO. Laparoscopic splenectomy despite the presence of a left ventricular assist device. Am Surg 2010;76:1306-8.

19. Samoukovic $G$, Vassiliou M, Giannetti N, et al. Laparoscopic splenectomy in a patient with a Heartmate( $\left({ }^{\circ}\right)$ II left ventricular assist device. J Laparoendosc Adv Surg Tech A 2011;21:535-8. http://dx.doi. org/10.1089/lap.2011.0169

20. Hoefnagel AL, Pasternak R, Curle AE, et al. Laparoscopic gastric bypass in a patient with an implanted left ventricular assist device. J Cardiothorac Vasc Anesth 2012;26:880-2. http://dx.doi.org/10.1053/i. jvca.2011.02.012

21. Sathishkumar $S$, Kodavatiganti R, Plummer $S$, et al. Perioperative management of a patient with an axial-flow rotary ventricular assist device for laparoscopic ileo-colectomy. J Anaesthesiol Clin Pharmacol 2012;28:101-5. http://dx.doi.org/10.4103/0970-9185.92456

22. Naitoh T, Morikawa T, Sakata N, et al. Emergency laparoscopic cholecystectomy for a patient with an implantable left ventricular assist device: report of a case. Surg Today 2013;43:313-6. http://dx.doi. org/10.1007/s00595-012-0241-6. Epub 2012 Jul 4.

Correspondence: Dr. Thomas McGregor, Assistant Professor, Section of Urology, Department of Surgery, University of Manitoba, AE101 - 820 Sherbrook St., Winnipeg, MB; tmcgregor@sbgh.mb.ca 